\title{
SpS1-SOFIA studies of stellar evolution
}

\author{
R. D. Gehrz ${ }^{1}$, E. E. Becklin ${ }^{2}$ and T. L. Roellig ${ }^{3}$ \\ ${ }^{1}$ Department of Astronomy, School of Physics and Astronomy, 116 Church Street, S. E., \\ University of Minnesota, Minneapolis, MN 55455, USA, email: gehrz@astro.umn.edu \\ ${ }^{2}$ Universities Space Research Association, NASA Ames Research Center, MS 211-3, Moffett \\ Field, CA 9403, USA, email: ebecklin@sofia.usra.edu \\ ${ }^{3}$ NASA Ames Research Center, MS 245-6, Moffett Field, CA 94035, USA, \\ email: Thomas.L.Roellig@nasa.gov
}

\section{Introduction}

The U.S./German Stratospheric Observatory for Infrared Astronomy (SOFIA, Figure 1) is a 2.5-meter infrared airborne telescope in a Boeing 747-SP flying in the stratosphere at altitudes as high as 45,000 feet where the atmospheric transmission averages $\geqslant 80 \%$ throughout the $0.3-1600$ $\mu \mathrm{m}$ spectral region. SOFIA's first-generation instruments include broadband imagers, moderate
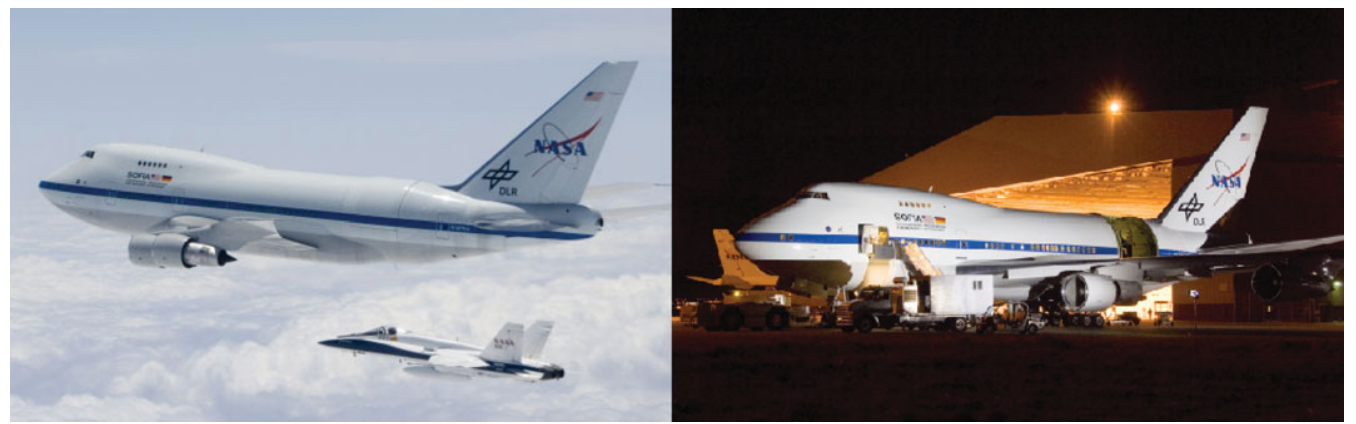

Figure 1. Left: SOFIA and F/A-18 safety chase plane during its second checkout flight on May 10, 2007. Right: Ground operations tests at Palmdale in March, 2008. NASA photos.

resolution spectrographs capable of resolving broad features due to dust and large molecules, and high resolution spectrometers suitable for kinematic studies of molecular and atomic gas lines at $\mathrm{km} \mathrm{s}^{-1}$ resolution. These and future instruments will enable SOFIA to make unique contributions to studies of the physics and chemistry of stellar evolution for many decades. Science flights will begin in 2010. A full operations schedule of at least 100 flights per year will begin in 2014 and will continue for 20 years. The SOFIA Guest Investigator (GI) program, open to investigators worldwide, will constitute the major portion of the SOFIA observing program.

\section{Spectroscopic studies of stellar evolution with SOFIA}

Stellar evolution in interstellar medium (ISM) plays a central role in the chemical evolution of the Universe (see Figure 5 in Becklin et al. 2007). New stars form from the ISM and produce heavy elements by main-sequence and explosive nucleosynthesis. The winds of postmain-sequence stars, novae, and supernovae inject processed material into the ISM, increasing its metallicity and therefore enriching the metal content of the next generations of stellar systems. A full understanding of the way that stars participate in this recycling requires studies of the physics and chemistry of all phases of stellar evolution from birth to death. SOFIA, with its wide wavelength coverage and high spectral resolution capabilities, is destined to play a dominant role in this field. Below, we give several specific examples of the role SOFIA will play in spectroscopic studies of various phases of stellar evolution. 


\section{SOFIA and regions of star formation in the ISM}

SOFIA's imaging instruments will provide detailed, broad/narrow band studies of regions of star formation to identify protostellar condensations and regions where the winds of embedded red giant stars, supergiant stars, and supernovae are interacting with the ISM. Follow-up studies with SOFIA's moderate and high resolution spectrometers will probe the detailed composition of the gas and dust, the physical dynamics, and other physical properties associated with these phases of stellar evolution. In particular, SOFIA can observe the atomic fine structure lines of [O I] at 63 and $145 \mu \mathrm{m}$ and of [C II] at $158 \mu \mathrm{m}$. These lines are bright in regions illuminated or shocked by the outflows of massive stars and supernova explosions. SOFIA's first light spectrometer (GREAT) will be unique in its ability to resolve these lines at the sub-km s${ }^{-1}$ level and probe in detail the physical conditions and kinematics in these regions.

\section{SOFIA and evolved stellar systems}

As an example or SOFIA's capability to study the late stages of stellar evolution, we consider nova explosions due to thermonuclear runaways caused by matter accreted onto white dwarfs in binary systems. Novae may contribute substantial amounts of isotopes of light metals to the ISM. IR observations can be used to quantify the physical parameters of novae explosions and assess their contribution to ISM chemical abundances (Gehrz 2008). Abundances in novae ejecta can be deduced from IR dust emission features and IR forbidden emission lines. Recent IR observations have shown that some novae have ejected shells that were extremely overabundant compared to solar values in $\mathrm{C}, \mathrm{N}, \mathrm{O}, \mathrm{Ne}, \mathrm{Mg}, \mathrm{Al}$, and $\mathrm{Si}$. The physical properties and mineralogy of dust produced by novae have been shown to be similar to those of the small grains in comets.

SOFIA will be a unique platform for observing novae because of its mobility and rapid deployability. First, monitoring of the temporal development of nova explosions requires observations occurring at all declinations and developing on time-scales of days, weeks, and months. Second, SOFIA's spectroscopic capabilities will enable the recording of many forbidden lines obscured by the atmosphere from ground-base observatories and heretofore unavailable to the spectrometers of other space missions. These lines are crucial for determining accurate elemental abundances in novae ejecta. SOFIA's spectrometers will provide a powerful probe of the physical conditions and kinematics of the ejected gas through studies of the atomic fine structure lines such as [O I] $(63,145 \mu \mathrm{m}),[\mathrm{O}$ III] $(52,88 \mu \mathrm{m}),[\mathrm{O} \mathrm{IV}](25.9 \mu \mathrm{m}),[\mathrm{C} \mathrm{II}](158 \mu \mathrm{m})$, [Si II] $(34 \mu \mathrm{m})$, and [S I] $(26 \mu \mathrm{m})$ and [S III] $(18.7 \mu \mathrm{m})$. Numerous forbidden neon lines in obscured spectral regions are also available to SOFIA. Neon is a major product of nova explosions.

\section{References}

Becklin, E. E., Tielens, A. G. G. M., Gehrz, R. D., \& Callis, H. H. S. 2007, Proc. SPIE 6678, 66780A-1

Gehrz, R. D. 2008, in Classical Novae, 2nd Edition, eds. M. F. Bode and A. Evans, Cambridge University Press: Cambridge, p. 167. 